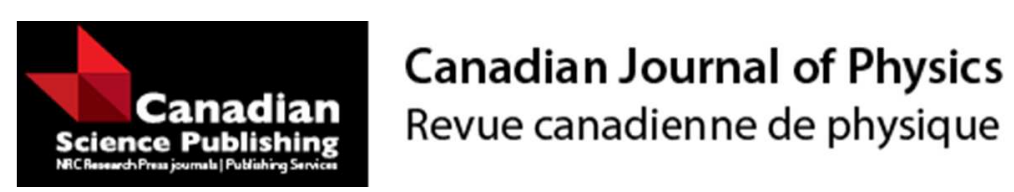

\title{
Parametric study of hyperfine structure of Zr II even-parity levels
}

\begin{tabular}{|r|l|}
\hline Journal: & Canadian Journal of Physics \\
\hline Manuscript ID & cjp-2016-0316.R2 \\
\hline Manuscript Type: & Article \\
\hline Date Submitted by the Author: & 29-Jul-2016 \\
\hline Complete List of Authors: & $\begin{array}{l}\text { Bouazza, Safa; Faculty of Sciences, Physics; University of Reims, Physics, } \\
\text { LISM }\end{array}$ \\
\hline Keyword: & $32.30 . J c, 32.10 . F n, 95.30 . K y, 35.10 . F k, 31.30 . J v$ \\
\hline \multicolumn{2}{|l}{} \\
\hline
\end{tabular}

SCHOLARONE $^{\text {Th }}$

Manuscripts 


\title{
Parametric study of hyperfine structure of Zr II even-parity levels
}

\author{
Safa BOUAZZA
}

Département de Physique, Université de Champagne-Ardenne, UFR SEN, BP 1039, F-51687 Reims

Cedex 2, France

Email: safa.bouazza@univ-reims.fr

\begin{abstract}
:
Up to now experimental hyperfine structure (hfs) data of twelve even-parity $\mathrm{Zr}$ II levels were given in literature. Recently new hyperfine splitting measurements of eleven other Zr II levels, of the same parity are achieved, applying fast-ion-beam laser-fluorescence spectroscopy. The hfs of these 23 gathered levels has been analysed by simultaneous parametrisation of the one-and two-body interactions, first in model space $(4 d+5 s)^{3}$ and secondly in extended one. For the three lowest configurations, radial parameters of the magnetic dipole $A$ and quadrupole electric $B$ factors are deduced in their entirety for ${ }^{91} \mathrm{ZrIl}$, compared and discussed with calculated values, available in literature and also with ours, computed by means of ab-initio method. For instance we give the main experimental values of the extracted single-electron hfs parameters of $4 d^{2} 5 s: a_{5 s}^{10}=-2701 \mathrm{MHz}$,

$\mathrm{a}_{4 d}^{01}=-122.4 \mathrm{MHz}$ and $\mathrm{b}_{4 d}^{02}=-113.5 \mathrm{MHz}$.
\end{abstract}

Keywords: Fine Structure, Hyperfine Structure, Landé-factors, Zr II spectrum and Ab-initio calculations.

PACS Nos.: 32.10.Fn, 31.30.Gs

\section{Introduction.}

The Zrll hyperfine splitting has been measured by Young et al. [1] and analysed theoretically by Beck and Datta[2] and by us[3]. We have to add furthermore that with help of on-line laser spectroscopy at the IGISOL facility in Jyväskylä the ionic ground state was studied by Thayer et al. [4]. Young et al., using the laser-rf double-resonance technics measured hyperfine splitting of Zrll levels arising exclusively from the metastable $4 d^{3}$ and $4 d^{2} 5 s$ configurations. For the first time hfs constants of the two only existing states of $4 \mathrm{~d} 5 \mathrm{~s}^{2}$ and also those of other new two lowest configuration levels are measured [5]. The complete details of the experiments are given for instance in [6].

We plan to extend in near future these experimental data to other ions of transition elements with an open $\mathrm{d}$ - and $\mathrm{p}$-sheels, like Moll for instance and to analyse their systems using a semi-empirical parameterisation method. Some hfs parameters of transition elements for $\mathrm{d}$ - and $\mathrm{p}$-shells are poorly evaluated up to now and it is interesting first to repertory their general trends. The big problem concerns mainly s-electron behaviour seeing its contribution to hfs A factor. What is exactly contactelectron impact and what is it its real interaction with nucleus? by what is it influenced when penetrating into the nucleus?

\section{Hfs considerations.}

Table 1 presents gathered experimental hfs data and for comparison we inserted computed hfs $A$ and $B$ factor values, done for angular momentum $\mathrm{J}=1 / 2$ and $\mathrm{J}=3 / 2$ by Beck and Datta [2] and also ours. We have two possibilities for semi-empirical analysis of the twenty three experimental data of table1, all concerning the three lowest configuration levels. The latter are close each others in energy and quite well isolated from the other configuration ones. For this reason it is better to study at first the model space $(4 d+5 s)^{3}$ and in the second step the extended model. Furthermore the model space generally 
permits to extract more accurate many-body parameter values.

For our hfs analysis we follow the many-body parameterisation method [7] which allows taking advantage of similarities between configuration interaction effects observed independently in spinorbit and hyperfine splitting. The radial parameters $\mathrm{a}_{n l}{ }_{n l}^{k}, \mathrm{~b}_{n l}{ }_{n l}^{k k}, \alpha_{\mathrm{i}}$ and $\beta_{\mathrm{i}}$ have been evaluated by fitting them to experimentally determined hfs constants $A$ and $B$ using the theoretical expressions (equations 4 and 5 of [8]).

The values of single-electron parameters, extracted by least square fitting procedure, quoted with their uncertainties, are presented in Table 2.

Usually these parameters are discussed for each configuration separately while in Table 2 some parameters are common to all configurations. The corresponding parameters for each configuration (Table 3) can be deduced from the model space parameters using:

$\mathrm{a}_{n l}^{k k}\left(\mathrm{I}^{\mathrm{N}+\mathrm{M}} \mathrm{s}^{2-\mathrm{M}}\right)=\mathrm{a}_{n l}^{k k}+[2 /(\mathrm{l}+1)][1-(\mathrm{N}+\mathrm{M})] \alpha_{1}-[2 /(\mathrm{l}+1)]^{1 / 2}(2-\mathrm{M}) \alpha_{4}+[2 /(\mathrm{l}+1)] \alpha_{5} \delta(\mathrm{M}, 0)$

for $\kappa \mathrm{K}=01,12 . \mathrm{In}$ the case of $\kappa \mathrm{K}=10$ one reads:

$\mathrm{a}_{n l}^{10}\left(\mathrm{l}^{\mathrm{N}} \mathrm{s}^{2}\right)=\mathrm{a}_{n l}^{10}$

$\mathrm{a}_{n l}^{10}\left(\mathrm{I}^{\mathrm{N}+1} \mathrm{~s}\right)=\mathrm{a}_{n l}^{10}-[2 /(\mathrm{l}+1)] \alpha_{9}$

$\mathrm{a}_{n l}^{10}\left(\mathrm{I}^{\mathrm{N}+2}\right)=\mathrm{a}_{n l}^{10}+[2 /(\mathrm{l}+1)]\left[\alpha_{10}-\alpha_{9}\right]$

Here $N=1$ and $M=0,1,2$. The values of $\alpha_{i}$ are given in Table 2 .

For our hfs analysis we had recourse also to extended model, composed from $4 d 5 s^{2}, 4 d^{2} 5 s, 4 d^{3}$, $4 d^{2} 6 s$ and $4 d^{2} 5 d$. The fs study was feasible except for experimental $4 d^{2} 5 d$ levels, given in literature, since some of them are really questionable.

For hfs analysis, model space and extended model display very close single-electron parameter values, but more accurate by means of model space, as expected. For this reason in Table 1 are reported only computed hfs constants obtained by this way. We give those obtained with the second path only in supplementary Table (Table S1) in order to insert hfs A and B of some $4 d^{2} 6$ s levels.

As in the case of odd-parity Zrll levels for configuration with open s-shell, i.e. $4 \mathrm{~d} 5 \mathrm{~s} 5 \mathrm{p}$, one parameter value is huge comparatively to other ones: $a_{5 s}^{10}\left(4 d^{2} 5 s\right)$. So our first reflex is to check its validity. One can note a fact that calculated ratio $a_{5 s}^{10} / g_{1}$ is in good interval in Table 4.

Let us try now to evaluate monoelectronic parameters for $\mathrm{s}$ - and d-electrons involved in this study:

for $5 s$-electron $\mathrm{a}_{5 s}^{10}=63.6145 \mathrm{~g}_{\mathrm{l}}<\mathrm{r}^{-3}>_{5 s}^{10}$

$$
=63.6145 \mathrm{~g}_{1} 4 \pi|\psi(0)|^{2} \text {. }
$$

with $g_{1}=\mu_{1} / \mathrm{I}=1.30362 / 2.5=0.521448$.

and for 4d-electrons we have:

$\mathrm{a}_{n l}{ }_{n l}^{k k}=2 \mu_{0} \mu_{\mathrm{B}} \mu_{1}\left(\zeta_{\exp } / \zeta_{\text {ab-initio }}\right)\left\langle\mathrm{r}^{-3}\right\rangle_{n l}^{k k} / 4 \pi \mathrm{l}=95.4128 \mathrm{~g}_{1}\left(\zeta_{\exp } / \zeta_{\text {ab-initio }}\right)\left\langle\mathrm{r}^{-3}\right\rangle_{n l}{ }_{n l}^{k k}$

with $\kappa \mathrm{K}=01,12,10$

$\mathrm{b}_{n l}^{\kappa k}=\frac{e^{2}}{h} \mathrm{Q}\left(\zeta_{\mathrm{exp}} / \zeta_{\mathrm{ab}-\text { initio }}\right)<\mathrm{r}^{-3}>_{n l}^{\kappa k}=234.9624 \mathrm{Q}\left(\zeta_{\text {exp }} / \zeta_{\text {ab-initio }}\right)<\mathrm{r}^{-3}>_{n l}^{\kappa k}$

with $\kappa \mathrm{K}=02,13,11$ and $\mathrm{Q}=-0.206$ barn 
$\zeta_{\text {exp and }} \zeta_{\text {ab-initio }}$ represent respectively spin-orbit constant values obtained experimentally and computed through ab-initio method [9]. We introduced the ratio $\zeta_{\text {exp }} / \zeta_{\text {ab-initio }}$ in order to weight expectation values found in many-body parameter relations. These corrections are very useful to get good agreements when p-electrons are particularly involved since $\zeta_{\text {exp }} / \zeta_{\text {ab-initio }}=$ around 1.4 generally. For d-electrons the impact of its introduction in (2) and (3) is less determinant since this ratio is around 0.92 (average value). In present study we will omit to use this ratio because we want to compare our data with those given in literature where this weight is not taken into account: multireference relativistic configuration-interaction [2] and relativistic sel-consistent-field calculations of the radial hyperfine integrals. The latter were done by Jonas R. Persson[10], using relativistic wavefunction of Hartree-Fock type and wavefunctions obtained by the so-called Optimised HartreeFock-Slater method [11].

We summarised in Table 5 expectation values obtained By Persson and those we obtained using pseudo-relativistic Hartree-Fock method [9]. Unfortunately we cannot use directly relation (1 twice) and $4 \pi|\psi(0)|^{2}$ value given in Table 5 of [3] because we need to scale the latter by a factor determined through experimental data of isotope shift [12].

We compare our extracted single-electron parameter values of Table 6 with those computed with aid of equations (1-3), through different ways. On the whole we have observed a good agreement between our extracted single-electron parameter values and those deduced from relativistic HartreeFock[10] or from our pseudo relativistic Hartree-Fock expectation values. Those computed from optimised Hartree-Fock seem overestimated.

Moreover we have noted previously that our extracted parameter $\mathrm{a}_{4 d}^{12}$ values for $\mathrm{Nb}$ II, Mo II and Ta II are smaller than $\mathrm{a}_{4 d}^{01}$. This trend is confirmed in present work, in total opposition with ab-initio calculations of Personn for a set of singly ionized atoms and those of Olsson and Rosen for a set of neutral atoms where systematically $\mathrm{a}_{4 d}^{12}$ values are bigger than $\mathrm{a}_{4 d}^{01}$ values. Dembczynski et al have confirmed our data trends when studying La II [13]. Let us indicate also that comparatively to experimental values contact- electron-d parameter $\mathrm{a}_{4 d}^{10}$ values are really under evaluated for the three configurations with ab-initio method even if $\mathrm{a}_{4 d}^{10}$ stands, in Table 5, only for relativistic effects, neglecting core polarisation contributions. In this latter Table $\mathrm{a}_{5 s}^{10}$ represent too only relativistic participation. So we have to add core polarisation contribution. For $\mathrm{Zr}$ I we have evaluated this contribution to $8.6 \%$. [14]. Once again, as for d-electrons, the method which gives good agreement between experimental and calculated $\mathrm{a}_{5 s}^{10}$ (when multiplying values given in Table 5 by a 1.086 factor) is Hartree-Fock one. The value obtained, using OHFS method seems on the other hand overestimated.

If we compare experimental A and B hfs constants given in Table 1 with those we computed, the agreement is excellent: we have only to point out that for level ${ }^{2} F_{7 / 2}$ of $4 d^{2} 5 s$, whose energy is: $6467.61 \mathrm{~cm}^{-1} \mathrm{hfs}$ constants A should be positive while B should be negative. On the other hand this comparison of experimental hfs data with those computed by Beck et al. [2], recurring to relativistic configuration-interaction approach is rather mitigated as in the cases of $\mathrm{Ti} / \mathrm{I}$ and $\mathrm{Hf} \mathrm{II}$. The methodology proposed by Beck consists in generating wave functions from Dirac-Coulomb Hamiltonian to which it is possible to insert Breit contribution, considered as a perturbation. Furthermore multiconfigurational Dirac-Fock solutions, extracted with help of Desclaux code [16] are used as zeroth-order functions.

\section{Conclusion.}

In this work we have taken advantage of new hfs measurements, very accurate, concerning for the first time $4 \mathrm{~d} 5 \mathrm{~s}^{2}$ configuration levels. All these experimental values are confirmed perfectly by our parametric calculations which are different from those given by Beck and Datta using three methods: Configuration Interaction (Cl), Dirack-Fock (DF) i.e. Relativistic Hartree-Fock and multiconfiguration 
relativistic configuration interaction (MRCl). This latter study is really courageous even if some clarifications are expected: $\mathrm{Zr}$ is considered as a light element. Do these codes, used in this study, give converging data also for high atomic numbers involving heavy elements? Why this study is limited only to two cases: $J=1 / 2$ and $J=3 / 2$ ? This work was extended [15] to $\mathrm{Zr}$ II Isoelectronic sequences: Hf II, Nb III, Ta III, Mo IV, W IV and La I. He showed that when using Dirac-Fock calculations one has to take into account both correlation and relativistic effects; otherwise calculated hfs constants may have wrong signs.

With more experimental data we have refined our previous parameter values given in [3] and generated predicted $A$ and $B$ hfs constant values for all known levels, inviting experimenters to extend the few existing measurements and hopefully to confirm our calculations.

\section{Appendix A: supplementary data}

The complete table of $A$ and $B$ hfs values sorted by angular momentum $J$ and increasing energy levels is presented in supplementary data associated with this paper.

\section{Acknowledgments}

We thank deeply Professors R. A. Holt and S. D. Rosner for helpful correspondence and for providing us their experimental data, prior to their publication 


\section{References}

1- Young L, Kurtz C A, Beck D R and Datta D 1993 Phys. Rev. A48 173

2- Beck D R and Datta D 1993 Phys. Rev. A48 182

3- Bouazza S 2011 Int. J. Quant. Chem 111: 3000

4- Thayer H L, Billowes J, Campbell P, Dendooven P, Flanagan K T, Forest D H, Griffith J R, Huikari J, Jokinen A, Moore R, Nieminen A, Tungate G, Zemlyanoi S and Äystö J 2003 J. Phys. G: Nucl. Part. Phys. 292247

5- Rosner S D and Holt R A 2016 J. Phys. B: At. Mol. Opt. Phys. 49115001

6- Holt R A and Rosner S D 2013 Hyperfine Interact. 216:21-26

7- Dembczynski J 1996 Phys. Scr. T 65, 88

8- Bouazza S, Dembczynski J, Stachowska E, Szawiola G and Ruczkowski J 1998

Eur. Phys. J. D 4, 39

9- Cowan R D TASS Berkeley University of California Press 1981.

10- Persson J R arXiv: 0704.3185vl [physics.atom-ph] 2008

11-Olsson G and Rosen A 1982 Phys. Scr. 26, 168

12- Aufmuth P, Heilig K, Steudel A 1987 At. Data Nucl Data Tables 37, 455

13 Dembczynski J, Elantkowska M,Furmann B and Ruczkowski J 2010 J. Phys. B

43, 065001

14- Bouazza S, Gough D S, Hannaford P and Wilson M 2000 J. Phys. B: At. Mol. Opt. Phys. 33 2355

15- Beck D R 1997 Int. J. Quant. Chem 65,555

16-Desclaux J P 1975 Comput. Phys. Commun. 9, 31

17- Ruczkowski J, Elantkowska M and Dembczynski J 2014 J. Quant. Spectrosc. Radiat.

Transf. 145:20-42

18- Bouazza S 2013 Phys. Scr. 87, 045301

19- Bouazza S, Holt R A, Rosner D S and Armstrong N M R 2014 Journal of Modern 
Physics, 5, 497

20- Bouazza S 2013 Phys. Scr. 87, 035302

21- Bouazza $S$ to be submitted

22- Bouazza S 2012 Phys. Scr. 86, 015302 


\section{Table Captions}

Table 1: Comparison between experimental and calculated hfs constants $\mathrm{A}(\mathrm{MHz})$ and $\mathrm{B}(\mathrm{MHz})$ of $\mathrm{Zr}$ II

Table 2: Fitted hfs parameter values $(\mathrm{MHz})$ for the model space $(4 \mathrm{~d}+5 \mathrm{~s})^{3}$ given with their standard deviations in parentheses.

Table 3: Fitted hfs parameter values (MHz) for each configuration under study.

Table 4: Variation of $\mathrm{a}_{n s}^{10} / \mathrm{g}_{\mathrm{I}}$ versus atomic number $\mathrm{Z}$ for some singly ionized atoms.

Table 5: Expectation radial integral values (a.u.) computed through different methods

Table 6: Comparison between extracted experimental hfs many-body parameter values (S.E.) and those computed by means of different methods. 


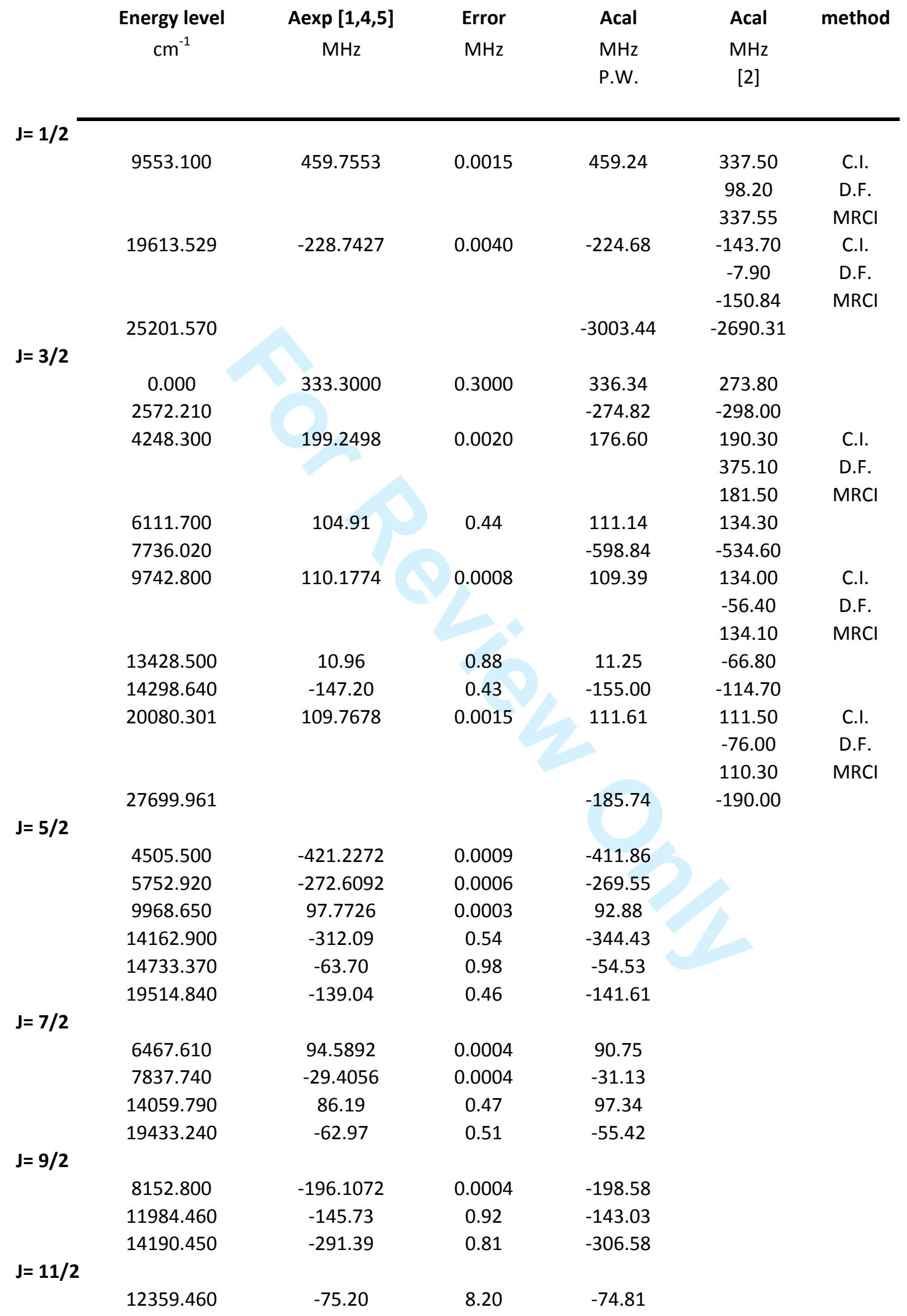


P.W.: present work C.I.: configuration interaction $\quad \mathrm{MRCl}$ : Multiconfiguration relativistic cor 


\begin{tabular}{|c|c|c|c|c|}
\hline $\begin{array}{c}B \exp [1,4,5] \\
\mathrm{MHz}\end{array}$ & $\begin{array}{l}\text { Error } \\
\mathrm{MHz}\end{array}$ & $\begin{array}{l}\text { Bcal } \\
\mathrm{MHz} \\
\text { P.W. }\end{array}$ & $\begin{array}{c}\text { Bcal } \\
\mathrm{MHz} \\
{[2]}\end{array}$ & method \\
\hline & & 0.00 & & \\
\hline & & 0.00 & & \\
\hline & & 0.00 & & \\
\hline-14.700 & 0.800 & $\begin{array}{r}-14.72 \\
14.46\end{array}$ & $\begin{array}{r}-12.46 \\
11.8\end{array}$ & \\
\hline 7.706 & 0.008 & 9.99 & $\begin{array}{r}5.93 \\
17.26 \\
5.82\end{array}$ & $\begin{array}{c}\text { C.I. } \\
\text { D.F. } \\
\text { MRCl }\end{array}$ \\
\hline 26.600 & 2.600 & $\begin{array}{c}18.89 \\
-35.83\end{array}$ & $\begin{array}{r}27.23 \\
-32.86\end{array}$ & \\
\hline 34.713 & 0.003 & 31.65 & $\begin{array}{l}27.46 \\
32.18 \\
27.46\end{array}$ & $\begin{array}{l}\text { C.I. } \\
\text { D.F. } \\
\text { MRCl }\end{array}$ \\
\hline-32.800 & 4.600 & -31.55 & -34.01 & \\
\hline 26.500 & 4.500 & 19.13 & 17.08 & \\
\hline 33.716 & 0.007 & -49.28 & $\begin{array}{r}28.51 \\
6.98 \\
28.3 \\
-46.14\end{array}$ & $\begin{array}{l}\text { C.I. } \\
\text { D.F. } \\
\mathrm{MRCl}\end{array}$ \\
\hline 8.195 & 0.011 & 8.92 & & \\
\hline-31.307 & 0.007 & -25.35 & & \\
\hline-42.969 & 0.004 & -38.95 & & \\
\hline-38.000 & 5.600 & -44.60 & & \\
\hline 34.000 & 20.000 & 21.61 & & \\
\hline-51.500 & 3.700 & -28.35 & & \\
\hline-39.878 & & -31.75 & & \\
\hline-60.181 & 0.007 & -48.44 & & \\
\hline-126.700 & 7.300 & -91.65 & & \\
\hline-47.300 & 6.300 & -27.64 & & \\
\hline-70.336 & 0.009 & -55.27 & & \\
\hline-59.000 & 20.000 & -79.65 & & \\
\hline-79.000 & 15.000 & -93.77 & & \\
\hline & & -85.52 & & \\
\hline
\end{tabular}


Ifiguration interaction D.F.: Dirac-Fock 
Table 2

\begin{tabular}{|l|c|}
\hline hfs parameter & Value $(\mathrm{MHz})$ \\
\hline $\mathrm{a}_{5 s}^{10}$ & $-2701.6 \pm 82.4$ \\
\hline $\mathrm{a}_{4 d}^{01}$ & $-139.7 \pm 4.6$ \\
\hline $\mathrm{a}_{4 d}^{12}$ & $-118.3 \pm 7.6$ \\
\hline $\mathrm{a}_{4 d}^{10}$ & $73.1 \pm 3.2$ \\
\hline $\mathrm{a}_{I C}^{12}$ & $-54.4 \pm 0.4$ \\
\hline$\alpha_{1}$ & $-43.3 \pm 7.9$ \\
\hline$\alpha_{2}$ & $-8.4 \pm 1.5$ \\
\hline$\alpha_{3}$ & $-5.6 \pm 1.0$ \\
\hline$\alpha_{9}$ & $-372.0 \pm 2.9$ \\
\hline$\alpha_{11}$ & $-1402.2 \pm 10.9$ \\
\hline$\alpha_{4}=\alpha_{5}=\alpha_{6}=\alpha_{7}=\alpha_{8}=\alpha_{10}$ & 0.00 (fixed) \\
\hline
\end{tabular}


Table 3

\begin{tabular}{|l|l|l|l|l|}
\hline Config. & $\mathrm{a}_{4 d}^{01}(\mathrm{MHz})$ & $\mathrm{a}_{4 d}^{12}(\mathrm{MHz})$ & $\mathrm{a}_{4 d}^{10}(\mathrm{MHz})$ & $\mathrm{a}_{5 s}^{10}(\mathrm{MHz})$ \\
\hline $4 \mathrm{~d} 5 \mathrm{~s}^{2}$ & -139.73 & -118.29 & 73.07 & \\
\hline $4 \mathrm{~d}^{2} 5 \mathrm{~s}$ & -122.41 & -103.63 & 221.8 & -2701.64 \\
\hline $4 \mathrm{~d}^{3}$ & -105.08 & -88.96 & 221.8 & \\
\hline
\end{tabular}


Table 4

\begin{tabular}{|c|c|c|c|}
\hline Ion & Configuration & $\mathrm{a}_{n s}^{10} / \mathrm{g}_{\mathrm{I}}\left(\mathrm{MHz} / \mu_{\mathrm{N}}\right)$ & $\mathrm{Z}$ \\
\hline Sc II [17] & $3 d^{1} 4 s$ & 2457.23 & 20 \\
\hline Ti II [18] & \multirow{6}{*}{$\begin{array}{l}3 d^{2} 4 s \\
3 d^{3} 4 s \\
4 d^{2} 5 s \\
4 d^{3} 5 s \\
4 d^{4} 5 s \\
5 d^{3} 6 s\end{array}$} & 2649.92 & 21 \\
\hline V II [19] & & 3072.58 & 22 \\
\hline $\mathrm{Zr} \mathrm{II}$ & & 5179.8 & 39 \\
\hline $\mathrm{Nb}$ II [20] & & 5952.20 & 40 \\
\hline Mo II [21] & & 6321.93 & 41 \\
\hline Ta II [22] & & 18387.08 & 72 \\
\hline
\end{tabular}


Table 5

\begin{tabular}{|l|l|l|l|l|l|l|l|l|}
\hline Config. & method & $\left\langle\mathrm{r}^{-3}\right\rangle_{4 d}^{01}$ & $\left\langle\mathrm{r}^{-3}\right\rangle_{4 d}^{12}$ & $\left\langle\mathrm{r}^{-3}\right\rangle_{4 d}^{10}$ & $\left\langle\mathrm{r}^{-3}\right\rangle_{5 s}^{10}$ & $\left\langle\mathrm{r}^{-3}\right\rangle_{4 d}^{02}$ & $\left\langle\mathrm{r}^{-3}\right\rangle_{4 d}^{13}$ & $\left\langle\mathrm{r}^{-3}\right\rangle_{4 d}^{11}$ \\
\hline $4 \mathrm{~d}^{2} 5 \mathrm{~s}$ & $\mathrm{HFR}(\mathrm{PW})$ & 2.457 & & & & 2.457 & & \\
\hline & $\mathrm{HF}[10]$ & 2.442 & 2.589 & -0.066 & 70.79 & 2.454 & 0.311 & -0.115 \\
\hline & OHFS[10] & 2.588 & 2.741 & -0.068 & 81.93 & 2.602 & 0.329 & -0.120 \\
\hline $4 \mathrm{~d}^{3}$ & $\mathrm{HFR}(\mathrm{PW})$ & 2.146 & & & & 2.146 & & \\
\hline & $\mathrm{HF}[10]$ & 2.117 & 2.251 & -0.060 & & 2.127 & 0.277 & -0.104 \\
\hline & OHFS[10] & 2.195 & 2.343 & -0.067 & & 2.206 & 0.301 & -0.114 \\
\hline $4 \mathrm{~d} 5 \mathrm{~s}^{2}$ & $\mathrm{HFR}(\mathrm{PW})$ & 2.788 & & & & 2.788 & & \\
\hline & $\mathrm{HF}[10]$ & 2.782 & 2.945 & -0.073 & & 2.796 & 0.349 & -0.113 \\
\hline & OHFS[10] & 3.014 & 3.172 & -0.070 & & 2.303 & 0.358 & -0.124 \\
\hline
\end{tabular}

PW : present work 
Table 6

\begin{tabular}{|l|l|l|l|l|l|l|l|}
\hline Config. & $\begin{array}{l}\mathrm{a}_{4 d}^{01} \\
\text { S.E. }\end{array}$ & $\begin{array}{l}\mathrm{a}_{4 d}^{01} \\
\mathrm{HFR}\end{array}$ & $\begin{array}{l}\mathrm{a}_{4 d}^{01} \\
\mathrm{HF}\end{array}$ & $\begin{array}{l}\mathrm{a}_{4 d}^{01} \\
\text { OHFS }\end{array}$ & $\begin{array}{l}\mathrm{a}_{4 d}^{12} \\
\mathrm{~S} . \mathrm{E} .\end{array}$ & $\begin{array}{l}\mathrm{a}_{4 d}^{12} \\
\mathrm{HF}\end{array}$ & $\begin{array}{l}\mathrm{a}_{4 d}^{12} \\
\text { OHFS }\end{array}$ \\
\hline $4 \mathrm{~d}^{2} 5 \mathrm{~s}$ & -118.9 & -122.24 & -121.97 & -128.76 & -103.63 & -128.81 & -136.37 \\
\hline $4 \mathrm{~d}^{3}$ & -103.8 & -106.77 & -105.33 & -109.21 & -88.96 & -111.99 & -116.57 \\
\hline $4 \mathrm{~d} 5 \mathrm{~s}^{2}$ & -139.9 & -138.71 & -138.41 & -149.95 & -118.29 & -146.52 & -157.82 \\
\hline
\end{tabular}

\begin{tabular}{|l|l|l|l|l|l|l|}
\hline Config. & $\begin{array}{l}\mathrm{a}_{4 d}^{10} \\
\text { S.E. }\end{array}$ & $\begin{array}{l}\mathrm{a}_{4 d}^{10} \\
\mathrm{HF}\end{array}$ & $\begin{array}{l}\mathrm{a}_{4 d}^{10} \\
\text { OHFS }\end{array}$ & $\begin{array}{l}\mathrm{a}_{5 s}^{10} \\
\text { S.E. }\end{array}$ & $\begin{array}{l}\mathrm{a}_{5 s}^{10} \\
\mathrm{HF}\end{array}$ & $\begin{array}{l}\mathrm{a}_{5 s}^{10} \\
\text { OHFS }\end{array}$ \\
\hline $4 \mathrm{~d}^{2} 5 \mathrm{~s}$ & 221.8 & 3.28 & 3.38 & -2701.64 & -2348.22 & -2717.75 \\
\hline $4 \mathrm{~d}^{3}$ & 221.8 & 2.98 & 3.33 & & & \\
\hline $4 \mathrm{~d} 5 \mathrm{~s}^{2}$ & 73.07 & 3.63 & 3.48 & & & \\
\hline
\end{tabular}

\begin{tabular}{|l|l|l|l|l|l|l|l|}
\hline Config. & $\mathbf{b}_{4 d}^{02}$ & $\mathbf{b}_{4 d}^{02}$ & $\mathbf{b}_{4 d}^{02}$ & $\mathbf{b}_{4 d}^{02}$ & $\mathbf{b}_{4 d}^{13}$ & $\mathbf{b}_{4 d}^{13}$ & $\mathbf{b}_{4 d}^{13}$ \\
& S.E. & HFR & HF & OHFS & S.E. & HF & OHFS \\
\hline $4 \mathrm{~d}^{2} 5 \mathrm{~s}$ & -113.50 & -118.9 & -118.77 & -125.94 & -17.82 & -15.05 & -15.92 \\
\hline $4 \mathrm{~d}^{3}$ & -99.01 & -103.9 & -102.95 & -106.77 & -15.66 & -13.40 & -14.57 \\
\hline $4 \mathrm{~d} 5 \mathrm{~s}^{2}$ & -125.99 & -135.0 & -135.33 & -159.87 & -20.43 & -16.89 & -17.33 \\
\hline
\end{tabular}

\begin{tabular}{|l|l|l|l|}
\hline Config. & $\begin{array}{l}\mathbf{b}_{4 d}^{11} \\
\text { S.E. }\end{array}$ & $\begin{array}{l}\mathbf{b}_{4 d}^{11} \\
\mathrm{HF}\end{array}$ & $\begin{array}{l}\mathbf{b}_{4 d}^{11} \\
\text { OHFS }\end{array}$ \\
\hline $4 \mathrm{~d}^{2} 5 \mathrm{~s}$ & 5.11 & 5.56 & 5.8 \\
\hline $4 \mathrm{~d}^{3}$ & 4.43 & 5.03 & 5.52 \\
\hline $4 \mathrm{~d} 5 \mathrm{~s}^{2}$ & 5.67 & 5.45 & 6.00 \\
\hline
\end{tabular}

S.E. : semi-empirical (experimental)

HF : relativistic Hartree-Fock

OHFS : optimized Hartree-Fock Slater

HFR : pseudo relativistic Hartree-Fock 